\title{
To curb COVID-19, global health must go local
}

\author{
The surge in COVID-19 cases in India and Brazil highlights the need to improve vaccine manufacturing capacity and \\ investment in public health at the local level.
}

T he impact of mass vaccination against COVID-19, with over one billion vaccine doses now administered worldwide, can already be felt in some countries, including Israel, the United Kingdom and the United States, where almost $50 \%$ of the total population has received at least one vaccine dose and cases have dropped in recent weeks. Alarmingly, the pandemic shows no signs of slowing down elsewhere-global COVID-19 cases in the last two weeks of April were higher than COVID-19 cases in the first six months of the pandemic, driven largely by surges in Brazil and India. The infection rates in these countries are at least partially due to low vaccination rates and the emergence of new, potentially more-transmissible, SARS-CoV-2 variants. Although high-income countries have provided aid to India, including shipments of personal protective equipment and oxygen, the dire situation in Brazil and India exposes both the importance of national leadership and the need for global assistance in the long term to improve local public-health infrastructure.

Leaders matter for public health. Brazilian president Jair Bolsonaro and former US president Donald Trump repeatedly ignored scientific evidence and downplayed the severity of the virus. Although Indian prime minister Narendra Modi initially seemed more willing to listen to scientific advisors than were his counterparts, he has prioritized large, maskless political rallies and a major religious festival over safety. Despite dysfunctional political leadership, both India and Brazil were widely expected to deploy vaccines against COVID-19 effectively, given their strong histories in organizing nationwide mass immunization campaigns. This has not been the case. At the time of this writing, only approximately $17 \%$ of people in Brazil and 10\% of people in India have received at least one vaccine dose. The case rates and poor vaccination coverage in these countries pose not only a regional problem but also a global roadblock for curtailing the pandemic, with the emergence of new variants.

The biggest problem facing the vaccination campaigns in both India and Brazil as well as other low- and middle-income countries (LMICs) is limited vaccine supply. India and Brazil did not purchase sufficient vaccines to immunize their populations. Ironically, data from the phase 3 trial of the AstraZeneca vaccine in Brazil were integral to its authorization by the European Medicines Agency, and India is one of the primary global manufacturers of vaccines. The Serum Institute of India, which is producing the AstraZeneca vaccine (called 'Covishield' in India), was supposed to supply India and export 200 million total doses for distribution via COVAX, an initiative for equitable access to vaccines against COVID-19, along with additional doses based on separate country-specific agreements. Expected shipments by the institute are now delayed, as the Indian government has blocked vaccine exports to shore up the domestic supply, further limiting availability elsewhere. Wealthier nations with greater purchasing power have ended up with vaccine surpluses capable of immunizing their populations multiple times over. Although some countries have already pledged to redistribute their vaccine stockpiles, and there is widespread public support for doing so, vaccine donations are now a matter of urgency. The unexpected recent backing from the US President Biden administration for temporary patent-protection waivers for vaccines against COVID-19, although a welcome move, will not immediately improve global vaccine supply, as the final text for a proposal still needs to be negotiated and approved by the World Trade Organization, which will probably take months. Moreover, the waiver on its own will not be sufficient even if and when it is formally approved. Although some LMICs, including Brazil and India, have the local capacity to manufacture vaccines, both the PfizerBioNTech vaccine and the Moderna vaccine require more-sophisticated manufacturing platforms than do traditional vaccines. It is notable that while Moderna stated in October 2020 that they would not enforce their patents, it appears that no third-party manufacturer has yet attempted to produce their mRNA vaccine. To be truly beneficial, an eventual intellectual property waiver will need to be accompanied by targeted aid to build both production infrastructure and robust pharmacosurveillance systems, along with the transfer of skills to ensure vaccine quality. Equitable vaccine distribution has to comprise both immediate redistribution of existing vaccine stockpiles and strengthening local vaccine manufacturing processes in LMICs to ensure that quality and efficacy are maintained.

Simply producing vaccines locally will not be enough. Vaccines are effective only when shots get into arms, and COVID-19 vaccine hesitancy in India and elsewhere will pose a problem even if supplies are eventually increased. It is essential to utilize local frontline healthcare and social workers, who are highly trusted by their communities and can therefore provide vital information about public health, and vaccine safety and efficacy. India, for example, has a program of Accredited Social Health Activists: female healthcare workers who play a critical role in managing the health needs of rural villages. In Brazil, the vacuum of reliable government communication has led to localized strategies-including businesses and social-media influencers-for disseminating information about vaccine availability and counteracting misinformation.

In addition, the global health community must look ahead to inevitable future outbreaks and pandemics. Beyond building local capacity for the production and distribution of new vaccines, it will be important to prioritize the evaluation of low-cost and easily produced vaccine options for LMICs. Local genomic surveillance and regional data sharing must also improve. Global cooperation in these areas, paired with national governance that invests in resilient public healthcare systems, will pay dividends both in the present and in long-term preparedness.

Without rapid engagement of high-income countries, the ongoing crises in India and Brazil are likely to be repeated in other LMICs, which will prolong the impact of the pandemic on everyone.

Published online: 24 May 2021

https://doi.org/10.1038/s41591-021-01400-y 